\title{
Title: Strengthening palliative care in the hospital setting: A co-design study
}

\section{Authorship team}

1. Ms Claudia Virdun - PhD Candidate and Senior Lecturer for the Faculty of Health, University of Technology Sydney (UTS); ORCID: 0000-0003-3945-0749

2. Dr Tim Luckett - Senior Lecturer for the Faculty of Health, UTS; ORCID: 0000-0001-6121-5409

3. Professor Patricia M Davidson - Dean of Johns Hopkins University School of Nursing, USA; Adjunct Professor of Nursing and Member of IMPACCT, UTS; Professor of Cardiovascular Research at St Vincent's Hospital Sydney;

4. Professor Jane Phillips - Professor Palliative Nursing and Director of IMPACCT (Improving Palliative, Aged and Chronic Care through Clinical Research and Translation), UTS; ORCID: 0000-0002-3691-8230

\section{Corresponding author contact details:}

Ms Claudia Virdun

Email: claudia.virdun@uts.edu.au

Mailing address: PO Box 123. Broadway NSW 2007 Australia

Telephone contact details: +61410437473

Word count: 4251 words

Key words: Palliative care, hospital, terminal care, consumer participation, patient-centred care 


\section{Acknowledgements}

The authors would like to acknowledge and thank each of the co-design workshop participants who so generously attended and contributed to the process and shaped our thinking. All authors contributed to the conceptualisation of this study and research protocol development. C.V led the recruitment and preparations for the workshop. J.P and C.V co-facilitated the workshop itself. C.V completed the initial data analysis prior to C.V, T.L and J.P participating in consensus discussions and worked together to inform the final set of recommendations presented. All authors contributed to the writing of this manuscript. This research is supported by an Australian Government Research Training Program Scholarship. This scholarship had no involvement in research design, conduct, manuscript preparation or choice of journal for submission. 


\section{Abstract}

\section{Objective:}

To identify actions required to strengthen the delivery of person and family centred hospital-based palliative care so that it addressed the domains of care identified as important for inpatients with palliative care needs and their families.

\section{Methods:}

A co-design study involving a workshop with palliative care and acute hospital policy, consumer and clinical representatives in Australia. A modified nominal group process generated a series of actions, which were thematically analysed and refined, before being circulated to participants to gain consensus.

\section{Results:}

More than half $(n=30,58 \%)$ of the invited representatives $(n=52)$ participated in the co-design process. Nine actions were identified as required to strengthen inpatient palliative care provision being: 1) evidence-informed practice and national benchmarking; 2) funding reforms; 3) securing executive level support; 4) mandatory clinical and ancillary education; 5) fostering greater community awareness; 6) policy reviews of care of the dying; 7) better integration of advance care planning; 8) strengthen nursing leadership; and 9) develop communities of practice for improving palliative care.

\section{Conclusions:}

Changes to policy, practice, education and further research are required to optimise palliative care within hospital settings, in accordance with the domains inpatients with palliative care needs and their families consider to be important. Achieving these changes will require a whole of sector approach and significant national and jurisdictional leadership. 


\section{Introduction}

Globally, there are considerable differences in cause of death and life expectancy, due to country of residence, socio-economic circumstances, lifestyle risks, ethnicity, gender and genetics. ${ }^{1}$ Low income countries report an overall life expectancy of 58.4 years, largely due to significant maternal and newborn deaths and deaths from communicable diseases. ${ }^{1}$ In contrast, high income countries have an overall life expectancy of 76.9 years, with the leading causes of death due to non-communicable diseases. ${ }^{1,2}$ It is projected that by 2030 the leading causes of death in high income countries will be: ischaemic heart disease, stroke, Alzheimer's disease and other dementias, lung cancer, lower respiratory infections, chronic obstructive pulmonary disease, colorectal cancers, diabetes, hypertensive heart disease and kidney disease. ${ }^{1}$

As a result of these epidemiological changes, the patterns of dying in high income countries have moved away from sudden deaths to the bulk of deaths being expected. Seminal work by Lynn and Adamson $(2003)^{3}$ has suggested that expected deaths can generally be classified as following one of three illness trajectories, namely: 1) a short period of evident decline (mostly cancer) where a person lives with illness, maintaining good function, followed by a reduction in this functioning, predominantly in the last few months of life; 2) long-term limitations with intermittent serious episodes (mostly organ failure) where a person lives with an overall decline in function, often over several years, with irregular acute episodes that contribute to ongoing functional decline; and 3) prolonged dwindling (mostly frailty and dementia) where people live with long-term functional decline and progressive disability and are at ongoing risk of dying from events such as infections, falls or fractures. Individuals following any one of these three trajectories can incur a significant symptom burden and have a commensurate need for palliative care. ${ }^{4}$ Advancing age and an increase in non-communicable diseases combine to confer complex health care needs and mean that an increasing proportion of people require care in the hospital setting, even if most would prefer to be cared for at home for as long as possible. ${ }^{5,6}$ Indeed, hospitals are an important care provider for people in their last year of life ${ }^{7}$ with benefits for palliative care reported for many such patients. ${ }^{8}$ Palliative care within this paper aligns with the definition from the World Health Organisation stating it is: 'an approach that improves the quality of life of patients and their families facing the problems associated with life-threatening illness, through the prevention and relief of suffering by means of early identification and impeccable assessment and treatment of pain and other problems, physical, psychosocial and spiritual. ${ }^{9}$ In line with this definition, this study asserts that palliative care is core business for all health care providers and specialist palliative care refers to care provided by clinicians whose substantive role is within palliative care..$^{10}$

For the last 30 years, patients and families have consistently reported the elements required for optimal inpatient palliative care with these being: Effective communication and shared decision making; Expert care; Adequate environment for care; Family involvement in providing care; Financial affairs; 
Maintenance of sense of self / identity; Minimising burden; Respectful and compassionate care; Trust and confidence in clinicians and Maintenance of patient safety and prevention of harm. ${ }^{7,11-14}$ However, delivering optimal, person-centred palliative care within acute, episodic care environments with varied access to specialist palliative care service support is challenging. Poor inter-disciplinary teamwork and limited communication combined with a lack of early identification of patients with palliative care needs all contribute to sub-optimal palliative care provision. ${ }^{15-20}$ Translating patient and family care priorities into actionable strategies is critical to drive the policy reforms required and enable clinicians and managers to implement best palliative care at the ward level.

Aim: To identify actions required to strengthen the delivery of person and family centred hospital-based palliative care so that it addressed the domains of care identified as important for inpatients with palliative care needs and their families.

\section{Methods}

\section{Design}

A co-design study was designed to derive a range of perspectives. Co-design describes how the experience of end users (patients and families) are used in combination with relevant stakeholders to inform service improvements. ${ }^{21-25}$ This study uses the following co-design definition: ${ }^{25}$

It involves patients and staff exploring the care pathway and the emotional journey patients experience along it, capturing experiences, then working together to understand these experiences and improve them (p.4). ${ }^{25}$

A number of prospective and systematic steps were undertaken to derive information that summarised the state of the science and questions to be posed at the workshop including: systematic reviews of patient and family data outlining what enables optimal inpatient palliative care; ${ }^{11,12}$ and a qualitative study to better understand published domains of importance, implications for practice and relevance for the Australian population. ${ }^{14}$ The outcomes from the meta-inference of these data ${ }^{26}$ formed the basis of the information packs for this co-design workshop ensuring end users (patients and families) remained central to all work achieved. This resulted in synthesised data representing what patients with palliative care needs $(n=1233)$ and their families $(n=3818)$ outlined as important for optimal hospital based palliative care ${ }^{26}$ (exploring the care pathway and capturing experiences) to enable those present at the workshop (clinicians, policy experts, researchers and consumer representatives) to co-design strategies to improve care experience based on this understanding.

\section{Participants}

Palliative care consumer representatives, identified professional leaders in hospital palliative care (either clinical or policy focused) or an identified academic leader working in palliative care research. Clinical 
leaders from medical, nursing and allied health professions were all eligible to participate. This study used the term 'palliative care consumer' to describe a person with palliative care experience (patient or family) supporting organisations in their work related to palliative care. Consumers were invited from two research centres focused on palliative care, one from a University setting and the other from a translational research network. Both these research centres enable meaningful consumer engagement through recruitment and training of palliative care consumers to support quality research.

\section{Setting}

In this study, 'hospital' is defined as all acute inpatient care excluding psychiatric, hospice or inpatient specialist palliative care, and alcohol and drug treatment centres. This study was undertaken in Australia where universal health coverage is available as well as considerable policy support for palliative care at national and jurisdictional levels. In addition to this policy environment, hospital care is governed by a national safety and quality accreditation system.

The co-design workshop (four hours) was held at an Australian University XXX (blinded for review) and facilitated by a member of the research team (C.V) who was supported by group facilitators who were external to the research team appointed within each working group.

\section{Recruitment}

Purposive sampling was used to identify eligible participants, given the need to ensure all participants were knowledgeable experts, to competently and efficiently inform study outcomes. ${ }^{27}$ This sampling approach also enabled recruitment reflective of all stakeholder groups eligible to participate. Each person was invited via email with two reminders, after which refusal was assumed. Interested participants were sent a participant information sheet with signed consent forms collected prior to workshop commencement.

\section{Data collection}

A modified nominal group process, ${ }^{28,} 29$ involving the following steps was used: an introductory presentation to frame the research context; individual idea generation; group discussion about generated ideas; group clarification of issues; group consensus on actions; workshop discussion and feedback; final pitch for a high priority action to ensure all ideas were captured; workshop conclusion (Figure 1). ${ }^{29}$ 


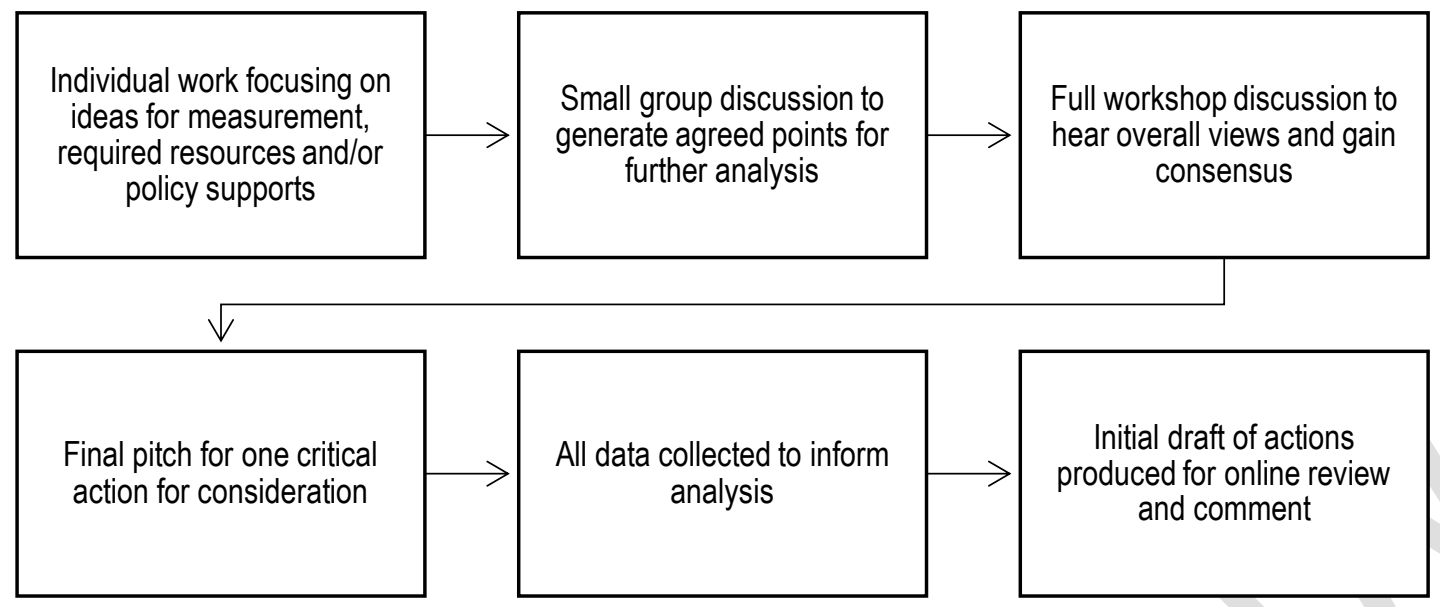

Figure 1: An overview of the modified nominal group process used

To facilitate the modified nominal group process, each participant was provided with an information pack detailing key domains of importance for optimal inpatient palliative care. ${ }^{11,12,14}$ Each participant was assigned to one of four groups designed to be reflective of all perspectives participating (medical, nursing, allied health, policy, academic and consumer). Three questions were provided to guide thinking and discussions:

1. If these practice points were working well - how would you know / what would it look like?

2. What needs to be in place to enable this to happen?

3. What policy changes are needed (if any) to make sure this happens?

The workshop was audio-recorded and notes taken. All collected data was analysed without any hierarchical assumptions being made. Theming completed following the workshop was circulated by email to all participants to enable their review and comment by a designated date.

\section{Reflexive account}

The co-design method adopted, inclusive of modified nominal group ${ }^{28,29}$ techniques, enhanced participation of all workshop attendees equally. An additional check was ensured by the appointing a priori of a group facilitator for each small group, drawn from the invited workshop participants, external to the research team.

\section{Data analysis}

The development of actions occurred through thematic analysis of collected data before circulating workshop outcomes to participants for feedback and relevant modifications. Thematic analysis was guided by the approach outlined by Thomas and Harden ${ }^{30}$ and included: 1) line by line coding (CV); 2) descriptive theme development (CV, TL and JP); and 3) analytical theme generation (CV, TL and JP). ${ }^{30,31}$ 
Analytical themes were presented as actions. Line by line coding occurred with the data collected on butchers' paper and data packs from each participating group. Where linkages within concepts were evident, these were grouped into broad descriptive themes ('actions') and discussed by the research team for consensus. These actions were circulated to participants (both those who attended and those who were unable to attend the workshop) for review and comment. Consensus was defined as response by a given date, with a non-response assumed to be agreement with circulated content. Where a response was obtained that required amendment to the first draft circulated, this was attended to and recirculated to the group accordingly. Where no further comments for review were obtained by a given date (with reminders provided) agreement with the final circulated content was assumed.

\section{Ethics approval}

Ethical approval was granted by St Vincent's Hospital Sydney, Human Research Ethics Committee Ref. No. 2019/ETH03307 in October 2019.

\section{Findings}

\section{Participants}

Key Australian palliative care and acute hospital policy, consumer, medicine, nursing or allied health representatives were identified and invited $(n=52)$, with $30(58 \%)$ attending. Of those who were unable to attend $(n=22)$ all contributed to the co-design process online. Details about the participant sample are provided in Table 1.

Table 1: Overview of the sample who attended the workshop and/or participated in targeted online feedback 


\begin{tabular}{|c|c|c|}
\hline Participant & Attended $(n=30)$ & $\begin{array}{l}\text { Apologies sent } \text { - included in online } \\
\text { communications }(n=22)\end{array}$ \\
\hline Senior medical palliative care clinicians & 5 & 3 \\
\hline Senior nursing palliative care clinicians & 6 & 2 \\
\hline $\begin{array}{l}\text { Senior palliative care allied health } \\
\text { representatives }\end{array}$ & 2 & 4 \\
\hline NSW state level policy experts & 6 & 6 \\
\hline National policy experts & 3 & 7 \\
\hline Palliative care consumer representatives & 4 & 0 \\
\hline Research team members & 4 & 0 \\
\hline
\end{tabular}

Participants were allocated into four inter-professional working groups (7-9 participants per group), specifically arranged to be inclusive of a medical, nursing, allied health, state and national policy expert, palliative care consumer and research representative. Given attendee numbers on the day, full coverage of all participant 'types' was not possible. However, each group was as diverse in roles as was feasible.

\section{Results}

Nine proposed actions, each with details of the steps required within these, were developed. These actions included: 1) evidence-informed practice and national benchmarking; 2) funding reforms; 3 ) securing executive level support; 4) mandatory clinical and ancillary education; 5) fostering greater community awareness; 6) policy reviews of care of the dying; 7) better integration of advance care planning; 8) strengthen nursing leadership; and 9) develop communities of practice for improving palliative care. A summary of the actions required at the macro (national), meso (organisation) and micro (ward/unit) levels, are summarised below, with the full results detailed in Appendix 1. Some actions inform suggested improvements at the ward level only (micro), the organisational level only (meso) or national level only (macro) whereas some involve suggested change across multiple levels as articulated below.

\section{Action 1: Evidence-informed practice and national benchmarking}

- Macro: Policy and infrastructure

Nationally, there is a need for the development of standardised patient and family reported experience measures, based on what is important for inpatients with palliative care needs, that can inform national benchmarking, highlight gaps in care and identify areas for improvement. Patient and family experience 
measurement is needed at two levels: 1) a higher-level focus (10-15 questions); and 2) a tool informing a 'deeper dive' if specific issues are identified. In addition, a predesigned pack to measure clinician and ancillary staff experience, morale and happiness at work is required. Streamlining access to available data was considered important, with recommendations made to: improve embedded coding for identification of patients coded as 'palliative' so data retrieval from current sources becomes possible (e.g. accessing data from inpatient experience surveys limited to people with palliative care needs); a national minimum data set for palliative care; and facilitated access to state and national level datasets.

- Meso: Resourcing and infrastructure

A process to inform optimal inpatient palliative care based on national measurement tools and indicators is required. Supporting senior clinical leadership at the ward level (via the nursing unit manager ('NUM') role) to redesign and innovate in response to such measurement is important. That is, organisational support, resourcing and infrastructure to enable clinicians to measure patient and family experience, receive timely feedback and innovate in response to this before measuring again, is required. Ensuring measurement of and response to clinician and ancillary staff experience, morale and happiness at work data is required. Finally, developing a process for reporting on and reviewing expected deaths in hospital within established Morbidity and Mortality meetings, including feeding this information back to the relevant NUM, will also support evidence-informed improvement work.

- Micro: Implementation and innovation

Strengthening inpatient palliative care based on the routine use of patient and family reported measures relies on local implementation of data collection and use. Ward-level nursing leadership, supported by national measurement tools and benchmarks will enable progression towards evidence-informed practice that is respectful of local approaches to care provision and innovations required.

\section{Action 2: Funding reforms}

- Macro: Policy

A review of the funding linked to inpatients coded as being 'palliative' is required. This coding should facilitate access to services needed to maintain comfort and function. Given patients clearly describe their wish to maintain independence where possible, facilitating rehabilitation supports is important. Finally, the need to ensure consistency in relation to palliative care resourcing and to actively resource inpatient palliative care beds in line with the Palliative Care Australia guidance, ${ }^{10}$ is highlighted.

\section{Action 3: Securing executive level support}

- Macro: Toolkit development

Development of a National Palliative Care Inpatient - Executive Engagement Toolkit ('toolkit') is required. It is suggested this work could be led by a national organisation, such as the Australian Commission on 
Safety and Quality in Health Care, to engage senior leadership and create incentives. The aims of this toolkit would be to: highlight what patients and families describe as most important for quality inpatient palliative care; and embed that inpatient palliative care is 'core business' and therefore strengthening the quality of this care, of paramount importance.

- Meso: Resourcing

Explicit resourcing to enable optimal inpatient palliative care is required. The foci of such resourcing includes: supporting NUMs with education and opportunities to lead collaborative improvement work; support for clinicians to spend time on reflective practices required to undertake this work; support for driving quality improvements through the identification and navigation of barriers; and developing a mechanism to reward excellence in care both to acknowledge the importance of hospital-based palliative care and to enable sharing of excellent practice.

\section{Action 4: Mandatory clinical and ancillary education}

- Macro: Policy and resource development

Ensuring a defined competency level of communication skill nationally, for clinicians and ancillary staff is required. Progressing from this, a national suite of learning resources for use both online and face-to-face is required and could be developed by a leading palliative care academic department. In addition, the need to co-design, with palliative care consumers, a learning package for hospital clinicians and ancillary staff that focuses on the need for kindness, gentleness, acknowledgment of personhood and human interaction, vital to the wellbeing of both patients and families requiring inpatient palliative care, is highlighted.

- Meso: Policy and implementation

Organisational leadership should articulate and resource minimum requirements for effective communication and shared decision-making competence for all clinicians and ancillary staff is required. Implementing annual mandatory training on effective communication, supported by the suite of evidencebased national learning resources, is essential. It is recommended that this education be disseminated at orientation and other appropriate fora.

\section{Action 5: Fostering greater community awareness}

- Macro: Policy

Adopting the success of the advocacy campaign in paediatric health highlighting the importance of 'the first 1000 days' of life was identified as an effective model, ${ }^{32}$ suggesting a campaign that promotes the last year of life as a further critical focus for healthcare. Co-designing this campaign with consumer representatives is critical. Avenues for promoting this campaign include: State and Federal government health ministers; national medical, nursing and allied health organisations; media outlets; and academic platforms. 


\section{Action 6: Policy reviews of care of the dying}

- Meso: Policy

Policy guiding the appropriate and respectful care for the recently deceased patient is required. The practice of applying identification tags to people who have died and placing them into body bags on the ward itself where this may cause distress to families, is questioned. It is suggested this procedure could be done instead, after removing the person from the ward.

\section{Action 7: Better integration of advance care planning}

- Meso: Infrastructure and policy

The need for infrastructure to enable the timely capture and access to advance care planning documentation within electronic medical record systems is essential to informing current care provision, inclusive of documented discussions, nominated substitute decision makers and agreed resuscitation status.

\section{Action 8: Strengthen nursing leadership}

Participants considered local hospital and ward level nursing leadership to be instrumental in enabling system level improvements in inpatient palliative care. They recommended using a co-design process to strengthen the NUM role to enhance palliative care provision given their ability to identify and navigate ward/unit level priorities. Equipping and supporting leadership and the operationalisation of personcentred care were seen to be critical.

\section{Leadership}

- Meso: Policy, resourcing and infrastructure

Leaders require support from their organisation to lead, drive and embed the profound cultural change required to ensure respectful and compassionate care is provided to every inpatient, including those with palliative care needs. Enabling a focus on relevant accreditation processes to embed systems and processes to enhance the safety and quality of care for inpatients with complex needs, is required.

- Micro: Implementation focus

Implementing changes led by the NUM, in line with local conditions, to enable care that aligns with patient and family identified areas of importance, is required. Supported by organisational policy, NUMs need to focus on driving evidence-informed change to ensure: all patients and families experience respectful and compassionate care; all clinicians and ancillary staff meet the stated minimal competence for effective communication and shared decision-making; and a process to enable a point of 'linkage' for information provision. Ensuring expert practice where nursing leaders confirm adequate skill-mix and that each clinician is working to the top of their scope of practice and is focused on the 'right' work for their role is important. 
Operationalising Person-centred care

- Macro: Resource development

The national collation of evidence-based tools that: facilitate routine and regular identification of inpatients with palliative care needs; facilitate optimal assessment and care planning; and operationalise personcentred care is required.

- Micro: Implementation focus

Implementation of the endorsed collation of tools, outlined above, is required. Ensuring the ward nursing team have the skills to ask patients directly about how to best support their maintenance of role, identity and meaning, and act on this information, is important. Optimising the ward environment, in line with areas of noted importance for patients, such as a quiet area and next to a window where possible, is another focus for ward-level nurse leadership. Nurses need to provide family members with an opportunity to be involved in the care of inpatients with palliative care needs. Ensuring that medical ward rounds occur at a set time, which is publicly available would facilitate more timely family input into care discussions.

\section{Action 9: Develop communities of practice for improving palliative care}

- Macro and Meso: Policy and resourcing

The implementation of local, regional and national inpatient palliative care communities of practice that engage ward level clinicians and specialist palliative care clinicians is important. These communities of practice aim to support the implementation of palliative care improvements, learning from experiences and sharing excellence.

\section{Discussion}

Findings from this co-design study yielded nine actions to enable hospitals to provide optimal inpatient palliative care. Embedded within these actions are a series of policy, practice, education and research recommendations at the macro, meso and micro levels. Given the complexity and diversity of palliative care needs and hospital environments (ranging from intensive, critical and high-dependency care units through to all ward areas and emergency departments), strengthening inpatient palliative care provision will benefit from: executive support declaring the importance of palliative care in hospitals; ${ }^{33}$ support for each ward to deliver care in accordance with patient and family identified areas of importance; ${ }^{11,12,14}$ integrated use of evidence-based tools; and validated and standardised approaches to measure patient and family outcomes and experiences to inform quality improvement, national benchmarking and ongoing models of care provision.

Enabling sustained improvements in palliative care within a system focused on the bio-medical approach looking to cure or prolong life is complex. ${ }^{17,19,20,34}$ Adapting key principles from the World Health 
Organization's Innovative Care for Chronic Conditions Framework ${ }^{35}$ may provide useful insights to guide clinicians, hospital administrators and policy makers. While this framework has been used widely in community settings, its components resonate strongly with the actions emerging from this study, including: a focus on placing patients and families at the centre of care; framing reforms within the context of a positive policy environment; the importance of a strategic reorganisation of healthcare (in this case, the ward environment) to deliver the required changes, and ensuring a well informed and engaged community. Addressing the changes required within each of these components would lead to improvements in line with this co-design studies' proposed actions (Refer Figure 2).

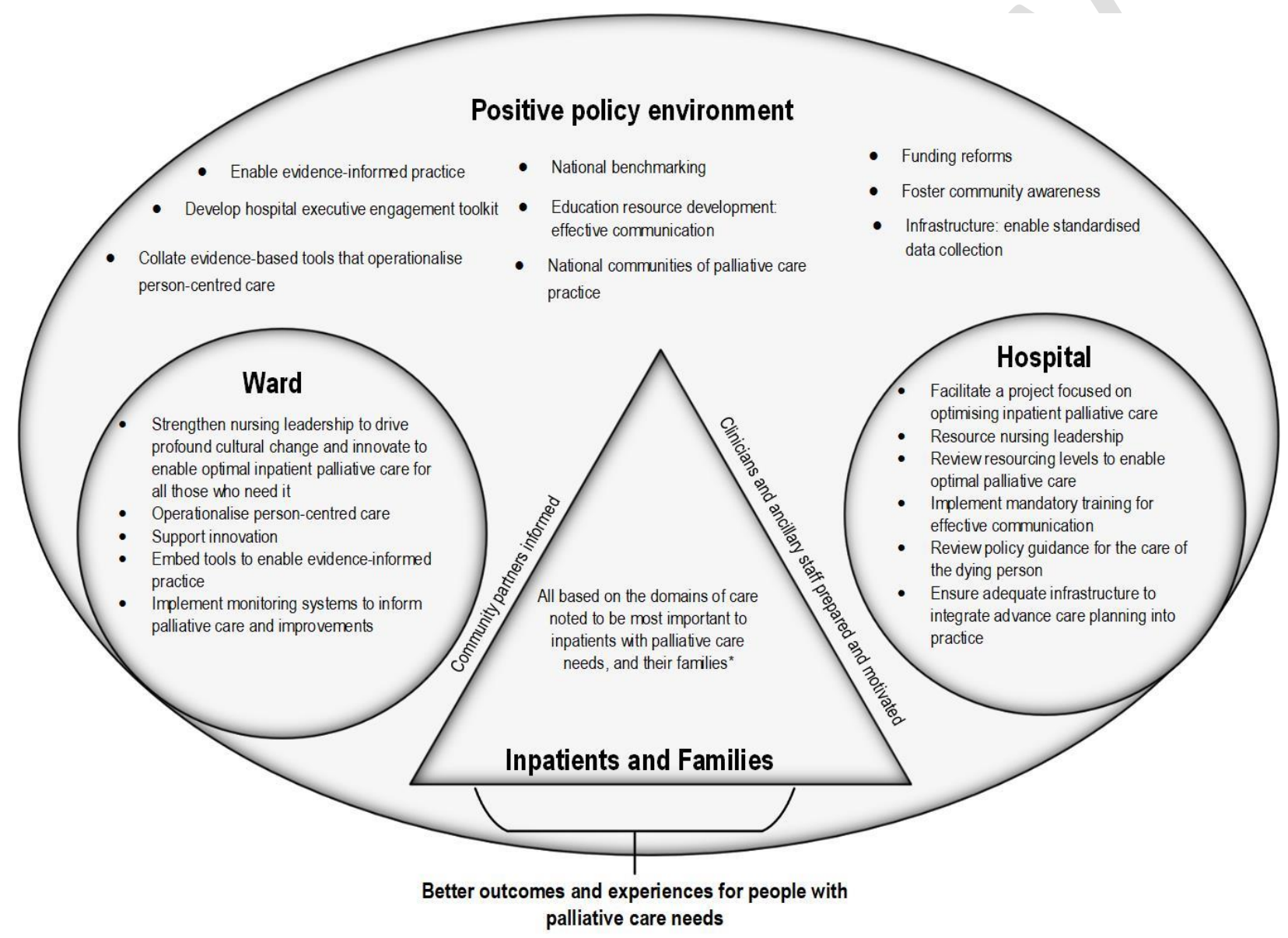

${ }^{*}$ Domains noted to be most important for optimal inpatient palliative care informed by previous research ${ }^{11,12,14}$

Figure 2: Adaptation of the Innovative Care for Chronic Conditions Framework ${ }^{35}$ to address the specific needs for the inpatient palliative care population. Innovative care for chronic conditions: building blocks for action: global report. Report no. 9241590173. Pruitt, S., \& Epping-Jordan, J. Chapter 3, Innovations in Care: Meeting the Challenge of Chronic Conditions, p.72 (2002). Access date - 29/04/20: https://www.who.int/diabetes/publications/icccreport/en/ (permission granted) 
There are many examples of excellent palliative care within the hospital setting. ${ }^{7,13,36}$ The challenge is to enable this experience to be possible across all wards, irrespective of location or population. Innovation at local levels (micro), supported by executive levels (meso) and networked to others working in similar settings is critical to reduce duplicated effort and identify the incremental changes that can contribute to improvements over time. ${ }^{37,38}$ To enable success, the next step in this work must be to work closely with clinical leaders to understand their perspectives, to work together to identify key areas of concern (informed by data where possible) and then allow their support whilst innovating to formulate solutions. ${ }^{37}$,

${ }^{39}$ The solutions to enabling optimal inpatient palliative care will be varied, albeit needing to be founded within available evidence. However, to enable successful commitment to change, clinical leaders will need to be drivers in both the discussion and implementation of changes. ${ }^{39}$ For example, one key area for analysis and innovation is in relation to accurate identification of people who have palliative care needs in the hospital setting. This is the first step in ensuring subsequent care planning and provision informed by the domains of optimal palliative care. ${ }^{11,12,14}$ There are several validated tools to assist in this work but how they will be implemented in each ward will vary. Imposing this process is neither helpful nor likely to lead to critical thinking or sustainable change. ${ }^{39,40}$ Therefore, starting with a group of clinical leaders to carefully consider the outcomes from this study is an important next step in progressing this work. In addition, the question of how to drive change in line with noted areas of importance is reliant on understanding how to measure such care to understand and evaluate change. How to do this in relation to which tools, datasets and indicators are feasible and acceptable requires attention. ${ }^{41}$ Better understanding data linkage opportunities is important both to inform clinical practice improvements as well as benchmarking and collaboration opportunities.

\section{Strengths and limitations}

This study's strength lies in its focus on patient and family perceived areas of importance for optimal inpatient palliative care from across the globe. ${ }^{11,12,14,26}$ Working from this foundation ensures the codesigned actions inform improvements in what matters most to those with palliative care needs. However, these data do not sufficiently reflect views of those from minority, culturally diverse and Indigenous communities and, therefore, it cannot be assumed that ensuing proposals would enable optimal inpatient palliative care experiences for all. It is noteworthy that the actions were co-designed with Australian palliative care consumers, policy experts and specialists in palliative care, rather than representatives from other specialties relevant to the bulk of hospital palliative care. It was deemed important to first seek advice from those with special expertise in palliative care within hospital settings to understand their perspectives on leading change. However, it is essential to also include perspectives from the broader hospital community in co-designing the next steps to ensure that interventions are acceptable and feasible for implementation and testing within their contexts of work. In addition, some of these actions 
may be transferable to settings outside of Australia but this will need careful review and consideration for varied local/national contexts working within different health system structures. In spite of the limitations, this is a critical step in leading system wide reforms.

\section{Conclusion}

A set of actions to inform changes to practice, policy, education and guide research are provided aimed at strengthening palliative care within the hospital setting. Importantly, these actions were co-designed with senior clinicians specialised in palliative care, policy experts and palliative care consumers and focused exclusively on domains of care identified as important by inpatients with palliative care needs and their families. The next step is to co-design interventions with hospital clinicians and administrators more broadly to enable implementation and testing of each action within clinical practice, so improvements are embedded and sustainable.

\section{Conflict of interest statement}

The authors declare no conflicts of interest.

Licence for Publication

The Corresponding Author has the right to grant on behalf of all authors and does grant on behalf of all authors, an exclusive licence (or non exclusive for government employees) on a worldwide basis to the BMJ Publishing Group Ltd to permit this article (if accepted) to be published in BMJ Supportive and Palliative Care and any other BMJPGL products and sublicences such use and exploit all subsidiary rights, as set out in our licence (http://group.bmj.com/products/journals/instructions-for-authors/licenceforms).

Competing Interest: None declared. 


\section{References}

1. Cherny NI, Fallon M, Kaasa S, et al. Oxford Textbook of Palliative Medicine. Oxford University Press, USA, 2015.

2. World Health Organization. The top 10 causes of death. 2018. Geneva: https://www.who.int/news-room/fact-sheets/detail/the-top-10causes-of-death (Accessed April 22, 2020).

3. Lynn J and Adamson DM. Living well at the end of life. Adapting health care to serious chronic illness in old age. 2003. United States: RAND Corporation

4. Knaul FM, Farmer PE, Krakauer EL, et al. Alleviating the access abyss in palliative care and pain relief-an imperative of universal health coverage: the Lancet Commission report. The Lancet 2018; 391: 13911454.

5. Gágyor I, Himmel W, Pierau A, et al. Dying at home or in the hospital? An observational study in German general practice. European Journal of General Practice 2016; 22: 9-15.

6. Heckel M, Vogt AR, Stiel S, et al. The quality of care of the dying in hospital-next-of-kin perspectives. Supportive Care in Cancer 2020: 1-11. 7. Gott M, Robinson J, Moeke-Maxwell T, et al. 'It was peaceful, it was beautiful': A qualitative study of family understandings of good end-of-life care in hospital for people dying in advanced age. Palliative Medicine 2019; 7: 793-801.

8. Robinson J, Gott M, Frey R, et al. Predictors of patient-related benefit, burden and feeling safe in relation to hospital admissions in palliative care: a cross-sectional survey. Palliative Medicine 2018; 32: 167171.

9. World Health Organization. WHO Definition of Palliative Care, http://www.who.int/cancer/palliative/definition/en/ (2014, accessed April 22 2020).

10. Palliative Care Australia. Palliative Care Service Development Guidelines. 2018. ACT: Palliative Care Australia.

11. Virdun C, Luckett T, Davidson PM, et al. Dying in the hospital setting: A systematic review of quantitative studies identifying the elements of endof-life care that patients and their families rank as being most important. Palliative Medicine 2015; 29: 774-796.

12. Virdun $\mathrm{C}$, Luckett $\mathrm{T}$, Lorenz $\mathrm{K}$, et al. Dying in the hospital setting: $\mathrm{A}$ meta-synthesis identifying the elements of end-of-life care that patients and their families describe as being important. Palliative Medicine 2016; 31: 587-601. 
13. Coimín DÓ, Prizeman G, Korn B, et al. Dying in acute hospitals: voices of bereaved relatives. BMC Palliative Care 2019; 18: 91.

14. Virdun C, Luckett T, Davidson P, et al. Hospital patients' perspectives on what is essential to enable optimal palliative care: a qualitative study.

Palliative Medicine 2020; https://doi.org/10.1177/0269216320947570.

15. Nevin M, Hynes $G$ and Smith V. Healthcare providers' views and experiences of non-specialist palliative care in hospitals: A qualitative systematic review and thematic synthesis. Palliative Medicine 2020; 34: 605-618.

16. Productivity Commission. Introducing competition and informed user choice into human services: reforms to human services, draft report. Canberra: Australian Government 2017.

17. Gardiner C, Cobb M, Gott M, et al. Barriers to providing palliative care for older people in acute hospitals. Age \& Ageing 2011; 40: 233-238.

18. Dalgaard KM, Bergenholtz $\mathrm{H}$, Nielsen ME, et al. Early integration of palliative care in hospitals: a systematic review on methods, barriers, and outcome. Palliative \& Supportive Care 2014; 12: 495-513.

19. Verhofstede R, Smets T, Cohen J, et al. End-of-life care and quality of dying in 23 acute geriatric hospital wards in Flanders, Belgium. Journal of Pain and Symptom Management 2017; 53: 693-702.

20. Bloomer MJ, Endacott R, O'Connor M, et al. The 'dis-ease'of dying: challenges in nursing care of the dying in the acute hospital setting. A qualitative observational study. Palliative Medicine 2013; 27: 757-764.

21. Borgstrom E and Barclay S. Experience-based design, co-design and experience-based co-design in palliative and end-of-life care. BMJ Supportive \& Palliative Care 2019; 9: 60-66.

22. Greenhalgh T, Jackson C, Shaw S, et al. Achieving research impact through co-creation in community-based health services: literature review and case study. The Milbank Quarterly 2016; 94: 392-429.

23. Eyles $\mathrm{H}$, Jull A, Dobson $\mathrm{R}$, et al. Co-design of mHealth delivered interventions: a systematic review to assess key methods and processes. Current Nutrition Reports 2016; 5: 160-167.

24. Bratteteig T BK, Dittrich Y, Holst Mogensen P, Simonsen J. International Handbook of Participatory Design. Routledge, 2012.

25. Boyd H, McKernon S, Mullin B, et al. Improving healthcare through the use of co-design. New Zealand Medical Journal 2012; 125: 76-87. 26. Virdun C, Luckett T, Davidson P, et al. Key areas of clinical practice that enable optimal palliative care in acute hospitals: a mixed methods study. Journal of Clinical Nursing 2020; Under review (Sept 22, 2020). 
27. Tongco MDC. Purposive sampling as a tool for informant selection. Ethnobotany Research and Applications 2007; 5: 147-158.

28. Allen J, Dyas J and Jones M. Building consensus in health care: a guide to using the nominal group technique. British Journal of Community Nursing 2004; 9: 110-114.

29. Wainwright $D$, Boichat $C$ and McCracken LM. Using the nominal group technique to engage people with chronic pain in health service development. The International Journal of Health Planning and Management 2014; 29: 52-69.

30. Thomas $\mathrm{J}$ and Harden A. Methods for the thematic synthesis of qualitative research in systematic reviews. BMC Medical Research Methodology 2008; 8: 45.

31. Popay J, Roberts H, Sowden A, et al. Guidance on the Conduct of Narrative Synthesis in Systematic Reviews. 2006.

32. Moore T, Arefadib N, Deery A, et al. The first thousand days: an evidence paper. Melbourne: Murdoch Children's Research Institute 2017. 33. Gott M and Robinson J. Are we getting it wrong? Perspectives on the future of palliative care in hospitals. Palliative Medicine 2018; 1: 2.

34. Phillips J, Halcomb E and Davidson P. End-of-Life Care Pathways in Acute and Hospice Care: An Integrative Review. Journal of Pain and Symptom Management 2011; 41: 940-955.

35. Pruitt S and Epping-Jordan J. Innovative care for chronic conditions: building blocks for action: global report. Report no. 9241590173, 2002. World Health Organization.

36. Robinson J, Gott M, Gardiner C, et al. A qualitative study exploring the benefits of hospital admissions from the perspectives of patients with palliative care needs. Palliative Medicine 2015; 29: 703-710.

37. Lukas CV, Holmes SK, Cohen AB, et al. Transformational change in health care systems: an organizational model. Health Care Management Review 2007; 32: 309-320.

38. Samuelsson P, Witell L, Gottfridsson P, et al. Incremental and radical service innovation in Healthcare. Handbook of Service Science, Volume II. Springer, 2019, pp.619-638.

39. Erlingsdottir G, Ersson A, Borell J, et al. Driving for successful change processes in healthcare by putting staff at the wheel. Journal of Health Organization and Management 2018; 32: 69-84.

40. Harvey G, Kelly J, Kitson A, et al. Leadership for evidence-based practice-Enforcing or enabling implementation? Collegian 2020; 27: 5762. 
41. Kamal AH, Bausewein C, Casarett DJ, et al. Standards, Guidelines, and Quality Measures for Successful Specialty Palliative Care Integration Into Oncology: Current Approaches and Future Directions. Journal of Clinical Oncology 2020; 38: 987-994. DOI: 10.1200/jco.18.02440. 